\title{
Corela
}

Cognition, représentation, langage

12-1 | 2014

Vol. $12, \mathrm{n}^{\circ} 1$

\section{Les stéréotypes de la femme dans la caricature de Dilem Ali}

Sonia Benamsili

\section{(2) OpenEdition}

Journals

Édition électronique

URL : http://journals.openedition.org/corela/3447

DOI : $10.4000 /$ corela.3447

ISSN : 1638-573X

Éditeur

Cercle linguistique du Centre et de l'Ouest - CerLICO

Référence électronique

Sonia Benamsili, « Les stéréotypes de la femme dans la caricature de Dilem Ali », Corela [En ligne],

12-1 | 2014, mis en ligne le 25 juin 2014, consulté le 19 avril 2019. URL : http://

journals.openedition.org/corela/3447 ; DOI : 10.4000/corela.3447

Ce document a été généré automatiquement le 19 avril 2019

\section{(c) (i) (2)(2)}

Corela - cognition, représentation, langage est mis à disposition selon les termes de la licence Creative Commons Attribution - Pas d'Utilisation Commerciale - Partage dans les Mêmes Conditions 4.0 International. 


\title{
Les stéréotypes de la femme dans la caricature de Dilem Ali
}

\author{
Sonia Benamsili
}

\section{Introduction}

1 La caricature est un genre de dessin humoristique qui interprète graphiquement un personnage ou une situation en mettant l'accent sur un détail, en focalisant le regard sur les singularités ou les défauts. Généralement donnée comme synonyme de charge, cette représentation comique se fonde sur la déformation, la transgression d'une situation, des caractéristiques d'un visage ou d'un corps :

«La caricature reposait volontiers sur une perturbation de la traditionnelle hiérarchie du corps. Les parties du visage comme les yeux, les oreilles et surtout le nez prenaient des aspects ambigus, la bouche se muait en orifice obscène. » (Baridon \& Guédron, 2006 : 92).

2 La presse écrite constitue le support par excellence de cet art graphique qui permet de souligner un problème, de parler de l'actualité du jour, de se moquer des hommes en place et de provoquer, même lorsque le sujet est tragique, le sourire du lecteur. La caricature se présente alors comme un acteur médiatique qui offre un lieu privilégié de rencontres culturelles, qui centre son intérêt sur l'actualité en présentant avec un minimum de mots et de traits une situation. En effet, selon Lochard \& Boyer (1998: 9), l'élaboration de la caricature repose sur les traits pertinents et une économie de moyens. Ces traits pertinents sont accentués à des fins de dérision et s'établissent comme des conventions graphiques qui permettent la reconnaissance du personnage et des situations représentées.

Disposant de peu de temps pour faire passer efficacement son message, la caricature se doit effectivement d'être brève, simple, rapide et exige des plans courts, frappants qui doivent être facilement compris par le grand public. Ces caractéristiques et ces exigences font de ce moyen de communication un terrain particulièrement propice aux stéréotypes, 
un genre fortement codé qui s'organise autour d'unités toutes faites qui cimentent le message.

En effet, le stéréotype a longtemps été considéré comme une forme figée réservée à l'usage ordinaire, voire à des journalistes pressés, et dénotait une vision rigide, simplifiée de la réalité, une "opinion toute faite, cliché, réduisant les singularités " (Rey, 2001: 718). Rieusset-Lemarié (1994 : 22) n'y voyait qu'« un facteur à la fois de banalisation et d'illusion sur la nature même du langage» et le qualifie de "crime contre l'humanité». Amossy \& Herschberg Pierrot (2007:5) parlent, quant à elles, d'« une hantise de la stéréotypie », en vue de dénoncer la production de masse en littérature et dans la presse. C'est dans ce sens que vont, jusqu'à nos jours, de nombreuses tentatives de définition du stéréotype (Lippmann 1964; Bardin 1980 ; Jahoda 1964 ; Morfaux 1980; Fischer 1996, etc.) dans laquelle les psychologues n'étaient pas loin de voir une réduction porteuse de préjugés, particulièrement de type raciste.

5 Cependant,

«Plutôt que de l'appréhender seulement comme reflet d'une subjectivité [...], le stéréotype mériterait également d'être abordé en termes d'efficacité, dans la mesure où il permet d'utiliser un savoir commun au service d'une narrativité minimale.» (Chemartin \& Dulac, $2005:$ 139)

6 Ainsi, les travaux récents de Amossy (1991), de Leyens (1996) ou de Dufays (1994) sur le stéréotype avaient ouvert cette voie que Amossy \& Herschberg Pierrot synthétisent dans leur ouvrage "Stéréotypes et clichés : langue, discours, société». En s'inspirant des théories de la psychologie sociale et de l'analyse linguistique, ces deux auteures tendent à relativiser ou neutraliser les aspects dévalorisants de ce phénomène et le présentent comme «l'objet d'une réhabilitation qui permet de souligner ses fonctions constructives» (2007:28).

7 L'intérêt accordé aux mécanismes cognitifs et culturels a permis de ne plus envisager le stéréotype de façon nécessairement péjorative. Son étude peut en effet désormais être d'ordre empirique (construction de l'identité sociale et de la cognition sociale). On y voit, selon Leyens « un processus qui vise à régler les interactions sociales avec la plus grande efficacité possible » (cité par Amossy \& Herschberg Pierrot, 2007 : 43), une démarche permettant de saisir l'information complexe venant de notre environnement et de la simplifier, afin de donner un sens au monde qui nous entoure.

8 Les travaux du philosophe américain Putnam (1975) sur la sémantique lexicale présentent, quant à eux, le stéréotype comme une partie de la signification qui répond à l'opinion courante associée au mot, comme une composante assurant une description du sens en usage, fondée sur une reconnaissance de la norme sociale et culturelle. C'est à partir de ces propositions de Putnam ainsi que de la théorie de la sémantique argumentative d'Anscombre \& Ducrot (1983) que Galatanu développe un modèle de description de la signification lexicale à trois strates: noyau, stéréotypes et possibles argumentatifs (SPA). Les stéréotypes sont alors envisagés comme une partie stable, « un ensemble ouvert d'associations des éléments [...] constituants des blocs d'argumentation interne " (Galatanu, 2009: 395).

9 Les linguistes et les psychologues redécouvrent donc l'intérêt de partager des sens communs pour construire un vivre-ensemble. Le stéréotype commence alors à perdre sa dimension péjorative, à redevenir un objet d'étude précisément dégagé des jugements souvent stéréotypés. 


\section{Problématique}

10 Le principal objectif du caricaturiste est d'accrocher l'attention du lecteur et de lui donner quelque chose de facile à lire. Pour cela, il a souvent recours à des stéréotypes. De nombreux domaines, groupes ou catégories de personnes se retrouvent ainsi touchés par ces images toutes faites, très enracinées dans l'imaginaire collectif.

11 Dans les caricatures qui constituent notre corpus de recherche (caricatures de Dilem Ali), les stéréotypes de la femme sont sans doute les plus présents. Ils sont les plus identifiables et les plus marqués. Celle-ci a en effet toujours séduit les dessinateurs et suscité chez eux un intérêt particulier : de tout temps, la femme est objet de fascination. On tente perpétuellement de percer le mystère qui l'entoure. Il est ainsi devenu habituel de la voir dans la publicité, dans les films, dans les caricatures, etc., dans des rôles stéréotypés. De ce fait, des travaux sur le stéréotype de la femme n'ont cessé d'augmenter : en linguistique, le lien entre genre et langage a intéressé des chercheurs en sociolinguistique (Labov 1966 ; Trudgill 1972). Leurs travaux ont été utilisés pour affirmer que le langage des femmes se distinguait de celui des hommes par ses nombreuses formes linguistiques de prestige. En psychologie (Williams \& Bennett 1975 ; Hall 1904), il était principalement question de répertorier les différents qualificatifs associés aux stéréotypes masculins et féminins. En didactique, on s'est essentiellement intéressé au stéréotype de la femme dans des manuels scolaires (Tisserant 2008; Crabbe 1985; Gaudreault 1977, etc.), et ce, dans le principal but de montrer à quel point l'image de la femme est stéréotypée et ainsi déconstruire les genres. Les sciences sociales, quant à elles, se sont penchées, non pas sur le caractère vrai ou faux des stéréotypes, mais sur la manière dont ils sont utilisés (leur utilité). Il n'est en effet plus question d'effectuer des vérifications toujours problématiques sur l'exactitude des schèmes collectifs figés, «la question de la véracité des stéréotypes se trouve largement dépassée» (Amossy \& Herschberg Pierrot, 2007 : 39), mais de voir comment le processus de stéréotypage affecte la vie sociale et l'interaction entre les groupes.

12 La présence abondante de stéréotypes féminins dans la caricature de Dilem Ali et l'envie d'expliquer leur fonctionnement et usage ont donc retenu notre attention et motivé notre recherche. Par ailleurs, le fait que l'image de la femme se retrouve travaillée, par le caricaturiste Dilem, de différentes manières, dans différents contextes a également suscité notre intérêt.

13 Les objectifs visés à travers cette étude sont les suivants :

14 -Voir comment l'image de la femme est utilisée et façonnée dans les caricatures.

15 - Montrer à quel point cette image est stéréotypée dans les messages caricaturaux.

16 - Démontrer la place et la fonction des stéréotypes féminins au sein des caricatures de Dilem Ali.

17 Ce travail vient donc répondre à une interrogation majeure qui est la suivante : Pourquoi et comment la femme est-elle utilisée dans la caricature de Dilem Ali ?

18 Ainsi, le stéréotype sera le point de départ, l'instrument d'étude et l'objet de cette étude. On va réfléchir à l'aide de cette notion et sur elle. 
19 Afin de répondre au mieux à cette problématique, nous présenterons dans un premier temps notre corpus ainsi que la méthode d'analyse, puis nous analyserons les stéréotypes féminins présents dans quelques caricatures de Dilem Ali.

\section{Corpus et méthode d'analyse}

Il existe de nombreux corpus permettant d'aborder et d'analyser les stéréotypes de la femme. Notre choix s'est porté sur les caricatures de Dilem Ali ; un dessinateur de presse écrite algérienne qui publie, depuis 1996, ses dessins dans le quotidien algérien d'expression française "Liberté », tiré à plus de 150.000 exemplaires. Les conditions de travail du caricaturiste Dilem sont très difficiles: pression journalistique, poursuites judiciaires pour outrage au chef du gouvernement algérien Abdelaziz Bouteflika et offense aux généraux, menace de mort par les groupes islamistes durant la guerre civile (entre 1990-1998). Son talent et le courage dont il fait preuve pour défendre ses valeurs sont largement reconnus à travers le monde (il a été récompensé par plus de 20 prix internationaux).

21 Si nous avons choisi ce caricaturiste, c'est pour ses dessins extrêmement riches qui traitent des sujets d'une multiplicité infinie et donc qui permettent de rattacher la femme à de nombreux thèmes (le travail, le foyer, la religion, la politique, la publicité, etc.) et ainsi aborder tous les stéréotypes qui lui sont rattachés.

Le corpus étudié est composé de 31 caricatures de Dilem Ali, nombre, à notre avis, assez suffisant pour répondre aux objectifs visés. Elles sont toutes parues dans une période allant de 2006 à 2010 ; durée qui nous a paru répondant, assez exhaustivement, aux besoins de notre analyse, à savoir examiner les multiples images de la femme, l'évolution de ce personnage et les rôles qu'elle tient dans la société.

23 Pour répondre à notre problématique, nous avons choisi de nous intéresser à quatre aspects de l'image de la femme présentés dans la caricature de Dilem Ali (un seul exemple est retenu par catégorie) : la femme en tant que ménagère ou mère, la femme en tant qu'objet, la femme musulmane et la femme qui travaille (indépendante). Le choix de ces catégories repose sur le fait que sur les 31 caricatures analysées, la femme est souvent dépeinte en fonction de ces quatre types figés.

24 Les stéréotypes étant « des ensembles organisés de relations entre des signifiants et des signifiés » (Dufays, 1994: 64), c'est dans le champ de la sémiotique que notre travail s'inscrit. Analyser des images c'est passer en effet par l'étude des signes. Cette approche nous permettra de :

25 -Considérer le mode de production du sens de la caricature, autrement dit la façon dont elle provoque des significations, des interprétations.

- Mettre en évidence tous les stéréotypes féminins présents sous une forme implicite ou explicite dans les caricatures de Dilem ainsi que leurs rôles. En effet, les stéréotypes se présentent rarement, dans le discours caricatural, sous une forme directe ou complète et ne se laissent pas figer dans des structures formelles repérables. Ils demandent presque toujours à être reconstruits par le lecteur. C'est à lui que revient la tâche (en s'appuyant sur ses connaissances linguistiques, encyclopédiques, mais aussi tout ce qui est connaissances préfabriquées) de rassembler, autour d'un thème, un ensemble de traits qui lui sont, d'habitude, attachés et ainsi repérer l'image attribuée à un groupe : «Si le 
stéréotype n'est pas présent avec tous ses attributs, le lecteur d'après ce qu'il connaît pourra compléter l'image de l'autre. » (Amossy, 2000 : 111).

Il s'agira pour nous, lors de cette analyse, d'énumérer dans un premier temps les choses que l'on voit: description, de haut en bas et de gauche à droite, des éléments textuels (titraille, paroles des personnages), iconiques (personnages, objets) et plastiques (couleurs, angle de prise de vue, échelle, etc.) de chaque caricature à l'aide de signifiants et de signifiés. La caricature sera, à cette étape de la lecture, simplement dénotée. C'est " le degré zéro de l'intelligible» (Adam \& Bonhomme, 1997 : 178). L'étape suivante, celle où la caricature est connotée, consistera à faire appel aux signifiés culturels pour construire un sens.

\section{La femme dans les caricatures de Dilem Ali}

\subsection{La femme ménagère}

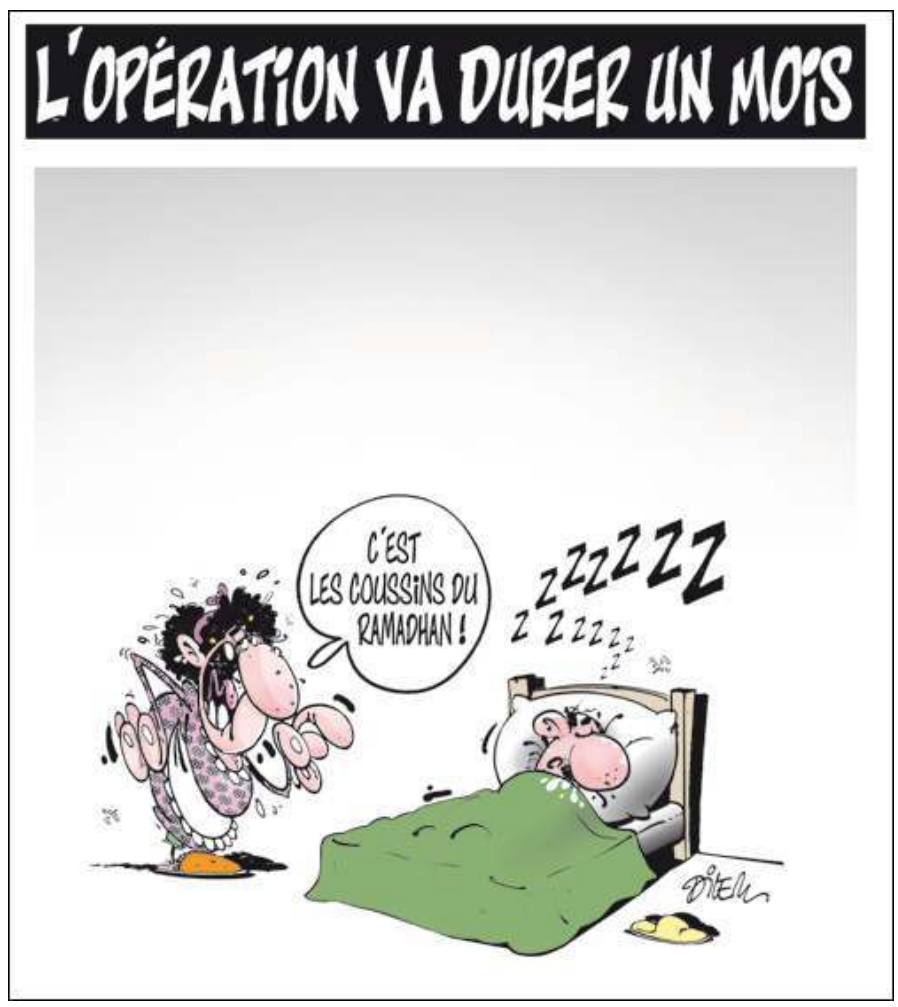

Caricature 1 : № 5461 du quotidien Liberté, dimanche 15 août 2010.

Apparait en haut de cette caricature une phrase verbale qui intitule ce dessin. L'image qui accompagne ce titre met en scène deux personnages types, un homme et une femme, vus de profil, dans un plan moyen. A gauche, la femme est représentée les traits du visage exagérés : nez énorme, bouche béante, longues oreilles. Elle porte une robe traditionnelle rose à fleurs, qui laisse entrevoir au-dessous un pantalon vert, et un tablier blanc. Un bout de tissu de même couleur que sa robe ramasse ses cheveux noirs et rebelles.

Main droite posée sur sa taille, cette femme, à l'apparence ridicule, guide le regard du lecteur en pointant de l'index gauche l'homme qui dort profondément dans son lit. Ses ronflements sont illustrés par la lettre « $\mathrm{z}$ » qui se retrouve multipliée, inscrite en gros caractères grandissants, lourds et noirs. 
30 La physionomie de cette femme, sa gestuelle ainsi que le point d'exclamation à la fin de son propos témoignent de sa colère et de son agacement. Elle prend la parole, regard dirigé vers le lecteur comme pour susciter sa pitié et l'impliquer dans la situation présentée. En effet,

«Cette vision donne l'impression que les personnages représentés s'adressent directement aux spectateurs ou aux lecteurs; elle a une fonction de contact [qui] capte obligatoirement l'attention du lecteur» (Cadet, Charles \& Gallus, 1990 : 20).

Les propos qui accompagnent ce premier personnage se caractérisent par le détournement d'une expression figée appartenant au langage familier et donc supposée connue de tous les lecteurs : "les couffins du Ramadan ». Expression qui fait référence aux dons accordés par l'Etat en ses multiples services et les bienfaiteurs, durant le mois de Ramadan, à une frange de la société exposée à la paupérisation.

Par ce détournement de référence culturelle, le caricaturiste séduit le lecteur grâce à une écriture pleinement ancrée dans son environnement discursif et culturel. En effet,

« Détourner est un jeu à tous points de vue : jeu pour les auteurs, jeu sur le langage, mais également jeu de lecture [...] Le lecteur est mis à contribution, il doit s'investir, creuser, chercher la source. » (Trudel, $2012: 20$ ).

Ce rapprochement paronymique «couffins»/ "coussins» lui permet également de compléter le comique iconique, de désambiguïser le lexème "opération » utilisé dans le titre, sans doute, pour capter l'attention du lecteur.

34 Associée à l'image, cette altération de forme et de contenu permet de mettre l'accent, d'un coté, sur la paresse et la lassitude qui se généralisent durant le mois sacré de Ramadan, qui est devenu au fil des années un mois de moindre travail, de quasi-inactivité, et, de l'autre, sur la relation stéréotypée de domination entre l'homme et la femme. En effet, ce jeu intertextuel ainsi que la représentation d'une femme avec son tablier typique du stéréotype de la femme au foyer, le dos courbé et les paupières tombantes, s'associent et forment un message informatif qui aborde, de manière implicite, un stéréotype d'ordre ethnique, à savoir celui de la femme arabe confinée aux occupations ayant trait au foyer et aux enfants. En effet, selon les traditions du peuple arabe, un seul rôle social est attribué à la femme, celui de ménagère, mère ou épouse. Ses activités restent ainsi liées au rôle traditionnel qui la relie à la maison. La séparation des mondes masculin et féminin marque donc les occupations du quotidien : les tâches ménagères sont du ressort de la femme, l'homme ne participe pas à la vie du foyer.

Le caricaturiste Dilem utilise ici ce stéréotype de femme au foyer comme argument en vue d'alerter les lecteurs sur l'inégalité de traitement, de répartition des rôles sociaux, domestiques entre l'homme et la femme. 


\subsection{La femme indépendante}

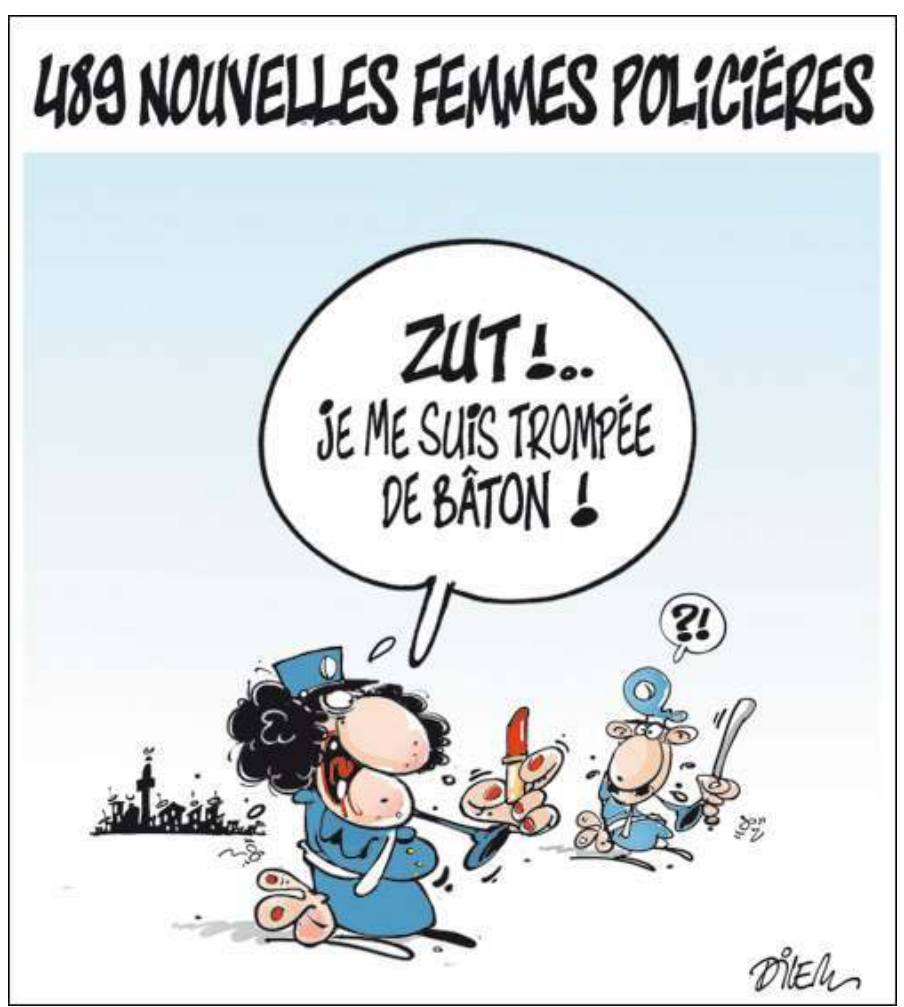

Caricature 2 : № 5555 du quotidien Liberté, dimanche 05 décembre 2010.

Le titre de cette caricature, par sa forme nominale et son vocabulaire neutre, est un titre informatif à caractère objectif. Il évoque le recrutement de 489 femmes dans le corps de la police. Au premier plan de l'image qui l'accompagne, apparait une femme, bâton de rouge à lèvres à la main gauche, vêtue d'un uniforme de police bleu et d'une casquette sur la tête qui laisse apparaître ses cheveux noirs et frisés. Vue de profil, son nez parait long et sa bouche grande ouverte. Elle porte un rouge à lèvres et du vernis aux ongles rouge ; couleur de passion et de séduction. Sur le coté gauche de ce personnage, au deuxième plan, apparait un homme lui aussi vêtu d'un uniforme de police. Il est vu de trois quart face, avec de longues oreilles et un long nez qui ne laisse entrevoir que sa moustache.

Ces deux personnages types sont représentés dans un plan moyen. Le minaret, la coupole de la mosquée, les maisons, les bâtiments, les paraboles sur les toits, tous ces éléments qui apparaissent à l'arrière plan indiquent qu'il s'agit d'une ville arabe, tandis que le fond bleu permet de situer la scène représentée dans un lieu ouvert (une rue).

Au-dessus de la tête du premier personnage apparait une grosse bulle centrée, occupant presque tout le haut de la caricature et véhiculant ses propos. L'interjection «Zut» de type exclamatif au début de cette phrase verbale permet de marquer le ton familier de cette discussion. Elle apparait en lettres épaisses assurant ainsi une fonction émotive : en effet, elle permet à la femme d'extérioriser, avec un son strident, son sentiment de surprise désagréable, de dépit, de déception et d'embarras quant à sa confusion entre le bâton de rouge à lèvres et celui de la police. La double ponctuation (point d'exclamation et de suspension) qui accompagne cette interjection, la goutte de sueur au-dessus de la tête de la femme et le point d'exclamation à la fin de sa phrase constituent une tentative 
de double transcription de la gêne et de l'irritation ressenties par cette femme ; émotion déjà prise en charge par l'interjection. L'homme quant à lui, regard tourné vers la femme, les yeux exorbités, est accompagné d'une petite bulle vidée de texte et renfermant une ponctuation doublée non conventionnelle qui marque l'incompréhension, l'inquiétude et l'étonnement de celui-ci.

Au-delà de son but informatif, le titre de cette caricature permet de mettre en place un nouveau stéréotype, celui de la femme moderne qui choisit d'être policière, d'entrer dans ce corps presque exclusivement masculin, soit par manque de travail et de besoin, soit par conviction (vocation) ou alors pour montrer sa force, son existence et sa responsabilité.

Le texte de la bulle et l'image de la femme s'associent quant à eux et se complètent pour délivrer une autre donnée informative : celle d'un stéréotype de genre qui vient confiner les femmes dans des rôles traditionnels bien précis (celui de mère, d'objet sexuel, de femme au foyer, etc.) car considérées incompétentes, faibles. Ainsi, "les femmes représentées sur leur lieu de travail [sont] rarement montrées en train de travailler " (Ceulemans \& Fauconnier, 1979: 10). Elles sont toujours vues à travers leur féminité, leur fécondité, leur moindre force physique. Ce stéréotype très présent dans la société arabo-musulmane fait que l'arrivée des femmes dans la police est souvent perçue comme une menace compromettant le travail (impossible de se reposer sur elles sur le terrain car jugées trop faibles), le statut social, l'image des hommes et les façons de faire: la rudesse, l'agressivité, etc., sont des compétences que les femmes ne possèdent pas car considérées trop émotives, sensibles et donc irrationnelles, incapables de maintenir une présence autoritaire dans des situations hostiles. En effet,

«Sur le plan psychologique, on estime que l'homme est plus enclin au sport, à la chasse et aux travaux physiques que la femme. Les sentiments de l'homme tendent vers le défi et le combat, alors que ceux de la femme tendent vers la paix. L'homme est agressif alors que la femme est relativement calme et tranquille. La femme a tendance à éviter la violence. » (Diagne, $2010: 23$ )

Par cette caricature, Dilem crée donc un nouveau stéréotype afin de rendre visible des modèles positifs, actifs, bref dominants de la femme. Et, en tournant la femme policière en dérision, en la ridiculisant, il dénonce tout simplement ces stéréotypes sexistes relatives aux différences sexuées dans des secteurs d'activités physiques et qui ne sont pas seulement produits par des différences de capacités naturelles entre les sexes, mais également par des croyances culturelles qui cherchent à maintenir la division sociale entre les hommes et les femmes, à conserver les femmes dans une position inférieure à celle des hommes. 


\subsection{La femme objet}

\section{DES MANNEQLIRNS AUI SALON DE L'ALITO}

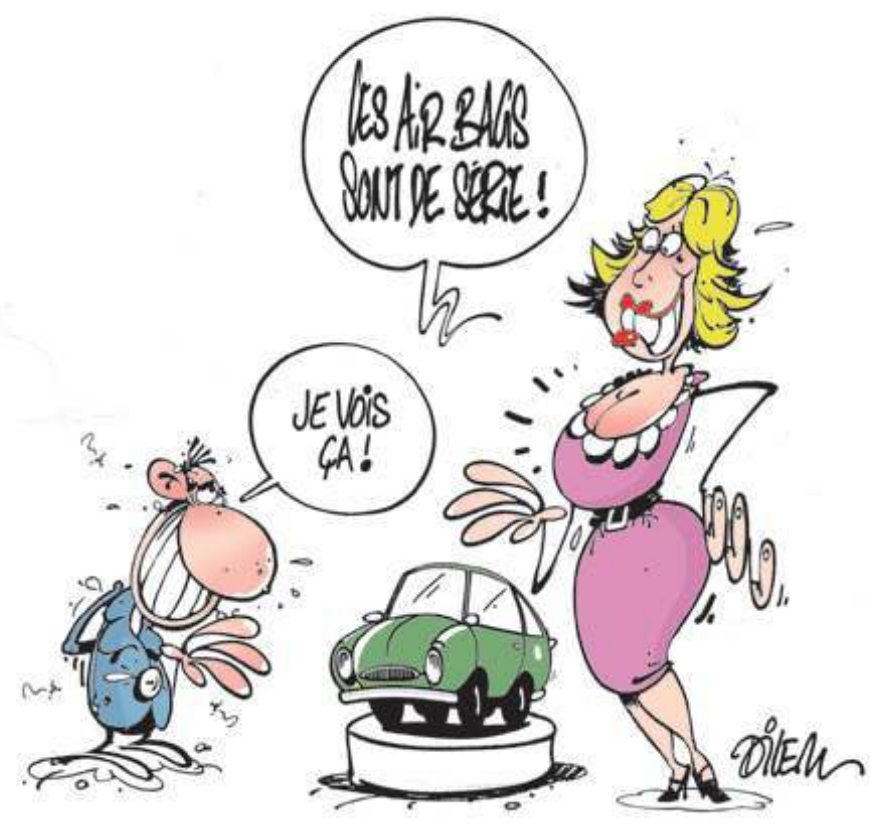

Caricature 3 : № 4731 du quotidien Liberté, mercredi 02 avril 2008. cette caricature qui met en scène deux personnages types, un homme et une femme, vus dans un plan moyen. A gauche, l'homme est représenté petit de taille, vêtu d'un costume bleu fermé par un seul bouton. Vu de profil, son nez parait gros et son oreille droite abimée. Main derrière le dos, il regarde, en souriant, la jolie femme blonde qui se trouve en face de lui. Celle-ci se tient debout sur des talons hauts, vêtue d'une robe moulante de couleur rose la mettant en valeur et accentuant sa beauté. Elle est représentée de trois quart, angle permettant d'accentuer ses formes généreuses, lèvres pulpeuses teintées en rouge et les cheveux bien coiffés. Elle pose sa main gauche sur la hanche, redressant ainsi naturellement son corps et acquérant une certaine prestance. Par l'autre main, elle désigne, avec un sourire commercial témoignant d'un état de réceptivité, d'ouverture et d'accueil, la voiture verte, à la forme arrondie et avec des courbes très féminines, placée dans l'axe du regard, pour en faire la publicité.

Cette caricature met donc en scène une publicité habituelle pour l'automobile où la présence de jolis mannequins, habillés de manière sexy et bien maquillés, sur les stands fait partie de la tradition. En effet, « les femmes représentés dans la pub sont toujours jeunes et séduisantes; elles sont fréquemment des objets sexuels» (Ceulemans \& Fauconnier, 1979: 14), car les femmes constituent le meilleur argument afin d'attirer l'œil des visiteurs, les inviter à se rapprocher et les inciter à l'achat: "Le but de la femme dans la vie est d'attirer» (Ibid.).

Cette image a par rapport au titre une fonction illustrative : les lexèmes « mannequins » et « auto » se profilent iconiquement dans la caricature. Associée aux textes des deux 
bulles qui accompagnent les personnages, elle ajoute des informations que le titre ne donne pas. En effet, cette image permet, par la présence d'un mannequin aux formes généreuses (stéréotype d'une femme blonde à forte poitrine) devant une voiture, par le regard et le sourire ironique et vicieux de l'homme qui semble visiblement plus intéressé par les courbes avantageuses du mannequin que par la voiture et par le lexème "airbags", utilisé ici comme un néologisme à connotation argotique, comme une création métaphorique désignant la poitrine d'une femme, de mettre en place le stéréotype réducteur de la femme reléguée au statut d'objet. Cette comparaison ou rapprochement (iconique et linguistique) entre les formes d'une femme et les courbes d'une voiture reflète en effet toute sorte de fantasmes masculins au détriment du respect de la femme.

Le message informatif de cette caricature met donc en valeur l'aspect sexuel du corps de la femme, présentée ici plus en tant que faire-valoir qu'en tant qu'acheteuse. Il la réduit à un instrument de séduction, à un objet de désir (appât) destiné à attirer le regard masculin vers le produit, à un être inférieur. L'objectif du caricaturiste, en ayant recours à ce stéréotype sexiste fortement ancré dans l'inconscient collectif (la relation femmevoiture), est d'illustrer, de manière parodique et sur le ton de la dérision, l'idée selon laquelle le monde de l'automobile reste un univers relativement machiste et que son ouverture aux femmes tient plus à une stratégie marketing qu'à un changement de mentalité d'une société patriarcale.

\subsection{La femme musulmane}

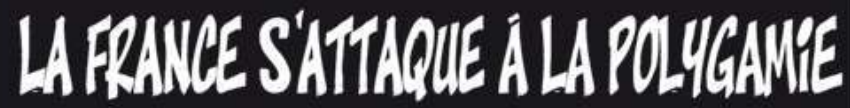

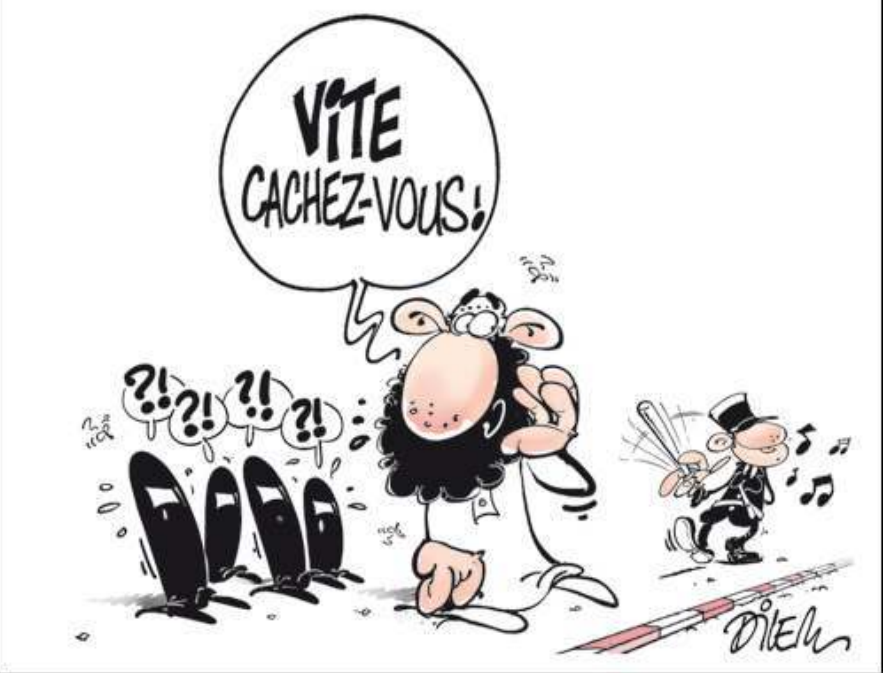

Caricature 4 : № 5457 du quotidien Liberté, mardi 10 août 2010.

Se trouve inscrit, en haut de cette caricature et dans une forme rectangulaire, un énoncé verbal informatif à construction syntaxique canonique (sujet + verbe + complément). Le 
verbe « attaquer » présent dans ce titre témoigne du rapport de tension existant entre la France, figure rhétorique d'ordre métonymique qui fait référence aux français, et la religion musulmane. Sans annoncer pourquoi la France s'attaque et s'oppose à la polygamie, l'information dans ce titre se présente comme une conclusion prédicative.

Apparait au premier plan de cette représentation un homme barbu, vu de trois quart, dans un plan moyen et portant des vêtements à l'islamique : une djellaba et une chéchia blanche sur la tête ; "couleur préférée du Prophète " (Mourregot, 2010: 84). Derrière ce personnage type, apparaissent quatre femmes; représentation visuelle du lexème "polygamie » présent dans le titre. Ce sont des personnages types dessinés en groupe. Elles apparaissent complètement voilées d'une burqa noire (couleur loin de constituer une parure et donc d'attirer le regard des hommes) et les yeux couverts par une bande blanche. Au loin, un troisième personnage, vêtu d'un uniforme de police, est représenté. Il avance, une matraque à sa main droite et l'autre réfugiée dans le dos, en chantonnant (les notes de musique qui l'accompagnent), dans la direction des autres personnages.

Le voile intégral des femmes ainsi que la djellaba et la barbe du premier personnage constituent des signes stéréotypés qui renvoient synecdochiquement à la culture arabe, à la religion musulmane. Ce sont des attributs de l'islam. Quant à l'emplacement de ces personnages (l'homme au premier plan et les femmes à l'arrière plan), la position des bulles qui les accompagnent ainsi que leurs tailles et contenus, ils permettent de traduire l'inégalité entre l'homme et la femme, de construire le stéréotype de l'oppression de l'homme et de la soumission de la femme dans la religion musulmane, sans doute dans le but de le dénoncer. Cette domination est également perçue dans le lexème "vite ", transcrit avec des lettres épaisses, l'usage de l'impératif et le point d'exclamation à la fin de l'énoncé verbal véhiculé par la première bulle. Ces signes traduisent en effet le son strident, le ton agressif et injonctif avec lequel l'homme s'adresse aux femmes qui se trouvent derrière lui. Celles-ci apparaissent d'ailleurs entourées de petits traits sous forme de gouttes de sueur; signes traduisant leur sentiment d'étonnement, leur état d'incompréhension et d'inquiétude. Elles sont accompagnées de bulles véhiculant un point d'exclamation "combiné avec le point d'interrogation pour indiquer à la fois surprise et intrigue» (Doppagne, 2006 : 37). Mais ces signes de ponctuations démontrent surtout que ces femmes sont mises aux baillons, muselées, réduites au silence. La burqa porte donc ici une signification de soumission de la femme qui dépasse sa portée religieuse.

L'enrichissement mutuel des deux constituants (linguistiques et iconiques) de cette caricature forme donc un message informatif qui permet de constituer l'isotopie / occident/, /orient/ très présente dans cette caricature et, de ce fait, de mettre en scène un stéréotype d'ordre ethnique qui renvoie aux pratiques religieuses qui font la différence d'un pays et qui constituent en dehors de ses frontières une image représentative, stéréotypée, empêchant l'intégration des personnes et créant des tensions interculturelles : les immigrés musulmans sont considérés en France, avec leurs mœurs, coutumes et religion, comme porteurs d'atteinte à l'identité nationale française. Les français voient dans la polygamie et le voile intégral un signe de la domination de l'homme sur la femme, une violence à l'égard des femmes et une négation de leurs droits à la dignité humaine.

Par cette caricature qui part d'une vision idéologique dépréciative toute faite de l'islam, le voile et la polygamie, le caricaturiste dénonce ces deux signes stéréotypés qui légalisent le contrôle exercé sur les femmes par les hommes, qui légitiment l'appropriation du corps des femmes. 


\section{Conclusion}

51 Au terme de cette étude, nous pouvons dire que de nombreuses représentations de la femme sont utilisées dans les caricatures de Dilem Ali. Les femmes ont en effet aujourd'hui des rôles variés dans la société. Elles composent avec des places parfois très différentes.

52 Les raisons qui amènent le caricaturiste Dilem, qui tente de toucher un public large, à faire appel à ces stéréotypes de la femme sont diverses. En effet, indépendamment des effets négatifs qu'il peut avoir, le stéréotype a une utilité importante. Il permet au caricaturiste de :

53 - Rendre visible l'invisible : l'enjeu essentiel du caricaturiste Dilem, en ayant recours à ces stéréotypes, ces connaissances partagées qui reflètent une image négative et réductrice des femmes, est d'interpeller les lecteurs. En effet, bien que la condition de la femme ait évolué, son image, elle, n'est pas utilisée de manière méliorative (elle reste très stéréotypée) et l'égalité entre les hommes et les femmes est loin d'être acquise.

54 - Révéler, par l'usage de ces stéréotypes réducteurs, les vices de la société.

55 - Déconstruire ces stéréotypes en les mettant en évidence ou bien en les contournant à l'aide de contre-stéréotypes, autrement dit en les retravaillant en vue de les modifier. En effet, comme le suggérait Flaubert, en 1880, dans Bouvard et Pécuchet « la seule façon de se défaire des stéréotypes est peut-être de les étaler au grand jour, d'en faire une matière première, quitte à les pousser jusqu'à l'absurde, pour mieux en rire» (cité par Chemarin \& Dulac, 2005 :159).

56 - Faire rire le lecteur: le caricaturiste utilise des stéréotypes, proposés sur un mode burlesque, afin de divertir ses lecteurs, de tourner en dérision certaines situations, certains personnages. Reste que ce rire n'est, dans la plupart du temps, pas innocent. En effet, derrière cet humour, le caricaturiste dénonce implicitement ces stéréotypes. Il cherche à accrocher l'attention du lecteur, le faire réagir.

57 L'usage du stéréotype s'avère être donc tout particulièrement pratique, stratégique. Il renvoie à l'idée d'un outil qui répond parfaitement aux exigences de production des caricatures, qui a toute sa place dans la création du message (contenu) que le caricaturiste cherche à faire passer, subrepticement, tout en l'introduisant derrière des banalités.

\section{BIBLIOGRAPHIE}

Adam, J.M., \& Bonhomme, M. (1997). L'argumentation publicitaire : Rhétorique de l'éloge et de la persuasion. Paris, Nathan.

Amossy, R. (2000). L'Argumentation dans le discours : Discours politique, littérature d'idées, fiction. Paris, Nathan. 
Amossy, R., Herschberg Pierrot, A. (2007). Stéréotypes et clichés : Langue, discours, société. Paris, Armand Colin.

Baridon, L., Guedron. M. (2006). L'art et l'histoire de la caricature : Des origines à nos jours. Paris, Citadelles \& Mazenod.

Cadet, C., Charles, R., Galus, J.L. (1990). La communication par l'image. Paris, Nathan.

Ceulemans, M., Fauconnier, G. (1979). Image, rôle et condition sociale de la femme dans les média. Etudes et documents d'information, 84. Paris, Unesco.

Chemartin, P., Dulac, N. (2005). La femme et le type : Le stéréotype comme vecteur narratif dans le cinéma des attractions. Cinémas : revue d'études cinématographiques, 16. 139-161. doi :

10.7202/013054ar

Diagne, M.H. (2010). La femme selon la vision islamique en Afrique noire. Paris, L'Harmattan.

Doppagne, A. (2006). La bonne ponctuation : Clarté, efficacité et précision de l'écrit. Bruxelles, De Boeck Supérieur.

Dufays, J.L. 1994. Stéréotype et lecture : Essai sur la réception littéraire. Liège : Mardaga.

Galatanu, O. (2009). Les incidences sémantiques des déploiements argumentatifs dépendants du co-(n) texte de production du discours. In E. Havu., J. Härmä., \& M. Helkkula (éds.),

Représentation du sens linguistique IV. Helsinki : Société Néophilologique. 391-405.

Lochard, G., Boyer, H. (1998). La communication médiatique. Paris, Seuil.

Mourregot, M.F. (2010). L'islam à l'île de la Réunion. Paris, L'Harmattan.

Rey, A. (2001). Le Grand Robert de la langue française. Paris, Le Robert.

Rieusset-Lemarié, I. (1994). Stéréotype ou reproduction de langage sans sujet. In A. Goulet (éd.), Le stéréotype : Crise et transformations (15-34). Caen, Presses Universitaires de Caen.

Trudel, E. (2012). Pratiques et enjeux du détournement dans le discours littéraire des XXe et XXIe siècles. Canada, Presses de l'Université du Québec.

\section{RÉSUMÉS}

La caricature de Dilem Ali puise dans un stock de représentations traditionnelles, de stéréotypes. Elle se nourrit de formes déjà connues pour communiquer rapidement son message et se faire comprendre par le plus grand nombre de lecteurs. Parmi ces stéréotypes, le plus présent est celui de la femme. Celle-ci se retrouve en effet représentée de différentes manières; rattachée à de nombreux thèmes. Elle constitue ainsi un terrain d'investigation propice permettant de voir la manière dont elle est utilisée dans les caricatures de Dilem, d'approfondir la notion de stéréotype, de démontrer l'importance de sa présence dans le discours caricatural ainsi que les différentes manipulations qu'en fait le caricaturiste pour produire ses dessins et atteindre ses objectifs. En d'autres termes, nous verrons comment la femme est représentée dans la caricature de Dilem Ali et nous dégagerons les objectifs du caricaturiste à travers cet usage.

The caricature of Ali Dilem is inspired from traditional representations and stereotypes. It feeds on forms already known to quickly communicate her message and to be understood by the largest number of readers. Among these stereotypes, women's one is the most used. Indeed, she is represented in different ways, and attached to many themes. Therefore, women are a perfect area of investigation to see how they are used in Dilem's caricatures, to deepen the concept of stereotype, to show the importance of their presence in the caricature and the caricaturist use of 
various manipulations to produce his drawings in order to achieve his objectives. In other words, we will see how women are represented in the caricatures and we will clarify Dilem's objectives through this use.

INDEX

Mots-clés : caricature, stéréotype, femme, sémiotique, signe, production, rôle du stéréotype Keywords : woman, semiotic, sign, role stereotype

\section{AUTEUR}

\section{SONIA BENAMSILI}

Doctorante, Université de Bejaia

benamsili86@gmail.com 